\title{
$\bullet$ \\ COVID-19 Pandemic: Psychological Stress, Ways for Patients to Cope, and Risk Mitigation
}

\section{IJCRR}

Section: Healthcare

ISI Impact Factor

(2019-20): 1.628

IC Value (2019): 90.81

$\operatorname{SJIF}(2020)=7.893$

\section{Rath N ${ }^{1}$, Sahai AK², Sood V ${ }^{3}$}

'Assistant Professor, Sri Sri University, Cuttack, Odisha, Cuttack- 754 oo6, India; ${ }^{2}$ Associate Professor, Sri Sri University, Cuttack, Odisha, Cuttack- 754 0o6, India; ${ }^{\mathrm{P}}$ rofessor, Sri Sri University, Cuttack, Odisha, Cuttack- 754 oo6, India.

\section{ABSTRACT}

The COVID 19 pandemic has an unprecedented effect on the world and has caused a huge death toll and miseries. It has manifest itself from disruption of normalcy to an economic slowdown and has impacted people in all walks of life. There is a growing body of evidence regarding a spike in depression and anxiety in the general public, due to the pandemic, and its negative impact on their daily lives due to death and economic hardships. It is not easy to handle the stress arising out of the pandemic and its ramifications. It is even more difficult for people with mental health challenges. The evidence has been pointing towards an increase in suicide in a high-risk population. The crisis is real, and efforts should be focused on ameliorating the risk in vulnerable populations. Patients need to be educated and trained to adopt lifestyle changes to deal with the rapidly evolving situation and restructure thought processes and utilize healthy coping skills, keeping them from slipping through the cracks. Communities and agencies need to work on strategies and policies that have been proven effective in preventing suicide and may have to come up with new measures to keep up with the emerging demands and challenges and work towards suicide prevention and zero suicide.

Key Words: Suicide, Risk prevention, COVID-19, Coping skills, Means restriction, Mental health

\section{INTRODUCTION}

We are currently going through stressful times, and there is no denying that the COVID-19 pandemic has rattled the entire world. It would be unimaginable to foresee any such calamity engulfing countries in such a short time and claim millions of lives unprecedented in human memory. This is an unprecedented crisis for the entire world and we are currently battling an invisible enemy that continues its relentless devastation.

With the progressive surge in the number of COVID-19 cases, it appears that the situation is here to stay. Along with the increased rate of depression and anxiety over this ongoing pandemic and the uncertainties associated with it, there is an increased vulnerability for suicide in those with mental health disorders. According to the Center for Disease Control (CDC) data, suicide is the $10^{\text {th }}$ leading cause of death in the United States and has been on a constant rise in the past decades. Although there is no updated 2020 statistics on suicide rates, the evidence suggests a surge in the number of suicides during the current pandemic, and there is data sup- porting increased suicides in previous pandemics. ${ }^{1}$ The risk for suicide increases 5-20-fold in patients with mental health disorders compared to individuals without mental health disorders. ${ }^{2}$ The presence of a life stressor is a precursor for suicide, and the current pandemic appears to have created the perfect triggers.

\section{THE IMPACT OF PANDEMIC}

The magnitude of the impact of COVID -19 is huge and farreaching. The COVID -19 restrictions and the limitations imposed to arrest the spread of the epidemic have resulted in untold miseries and hardships in our day-to-day lives. The economic chaos and the resultant job losses, salary cuts and furloughs, closing of schools, mandates for working from home, travel restrictions and bans, limitations on social gatherings, shutting down of the entertainment industry, and restricted freedom of movement.

People have varying levels of endurance in the face of stress. It is quite normal and commonplace for anyone to feel the

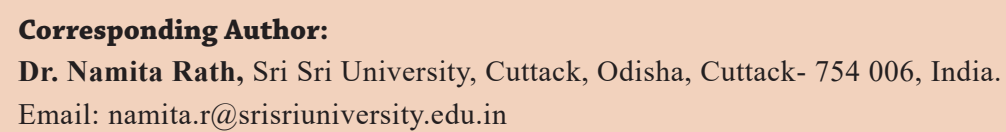


anxiety, frustration, and helplessness in such situations. Those afflicted by any mental health issues are more likely to give in and buckle under pressure. For such people, the uncertainty has been causing an overwhelming sense of fear and exasperation. The quarantine and social distancing have created isolation and lack of opportunities to stay active. Unemployment has taken a huge toll on the financial stability of the families who live on daily wage earnings. Falling behind on rent and bills, not being able to afford the basic needs, has created a state of distress on individuals who are already struggling. Those working from home are having to make several adjustments, creating further stress. The home environment may not be conducive to handle work from home, and the resultant changes and transitions may fall heavily on those with mental health issues. One must try to balance the work-life with the home front, such as managing an online school for kids and one's work from home. When a routine is disrupted and transitioning to a new norm, it can put a lot of strain on an individual and overwhelm them, making them easily succumb to pressure.

Available data suggests an upward tick in domestic violence, and alcohol consumption, which may exacerbate suicidal tendencies ${ }^{1}$. Individuals with mental health disorders, medical professionals, law enforcement, and front-line health care workers are in constant fear of possible exposure to viruses and succumbing to the illness. Working in a high-stress environment for hours and coming back to their family, with fear of the possibility of contracting COVID-19 and stressing about passing it on to their family members, takes a toll on the emotional stability of individuals that are already fragile. In the times where having job security is considered being fortunate, what does one do? Put up with the stress of the job, fear, and continue working? In India, hundreds of trained doctors and policemen have succumbed to Corona infections in the last six months.

Those living in remote places, with minimal access to health care/mental health services, and technology that allows access to services remotely, are being deprived of the services, leading to a lack of continuity of care. Patients needing help with their suicidal tendencies, may not visit ER, with the worry about contracting the virus, if they visit ER. Allencompassing, there is restricted access to services or places, they previously had. For some patients, meeting with their family members or going out, are the only means of entertainment, which the COVID-19 pandemic seems to have put an end to.

Are there changes patients can adapt to keep themselves from getting overwhelmed to the point of giving up? From restructuring one's thought process to making lifestyle changes and adjustments to the routine, there are several changes one can make, which could potentially alleviate the negativity caused by the stress and anxiety and help in finding a solution and a renewed push to move on with life instead of staying stuck in the negative emotions.

\section{COPING WITH STRESSFUL SITUATIONS}

Increased Awareness: The most important first step is acknowledging the problem and having a positive attitude to find a solution instead of fleeing away. This surely is a stressful situation; however, thinking on the terms of whether one has control over the current situation, and if they can change the reality, would help in accepting the experience nonjudgmentally. One must accept the situation as presented as inevitable and given. Focusing on things that we have control over, rather than worrying about situations that we have no control over is crucial to moving positively. Developing mindfulness and advanced consciousness through breathing techniques and meditation have been proven very useful in relieving stress. Yoga, meditation, and spirituality have helped many to live in the present moment and see the situation from a different perspective which takes away the stress caused by the immediate events. The negativity is removed by mindfulness, which is nothing but being aware of the present moment. Mindfulness helps gain back the stability and equilibrium of mind. Acceptance has been proven to be effective in ameliorating the causes leading to suicidal behaviors. $^{3}$

Thought restructuring: Finding an opportunity in the face of adversity. Instead of considering the current situation as a burden, those not working can improve their lifestyle. It is important to channelize energy productively and stay active. Making positive affirmations such as telling oneself, that this is not going to last forever, and it is just a matter of time to keep going.

Establishing a routine and having a structure to your day: We sure don't have control over the outside world, but we can control our timetable and make a routine. A systematic way of life reduces uncertainty and gives a feeling of being in the driving seat with control. Having a sleepwake routine, taking a shower in the morning, dressing up like going to the office, rather than just staying in pyjamas. These practices could benefit individuals. ${ }^{4}$

Getting enough sunlight: Research has proven the importance of sunlight. Opening blinds, pulling up curtains, and letting fresh air flow in are worthwhile. Exposure to sunlight is thought to increase Serotonin's release, a happiness hormone that alleviates the mood and brings calmness. A healthy, nutritious and balanced diet is also important with ample rest as this will boost immunity and also help maintain the mental balance.

Limiting media news exposure: It is important to keep the media exposure to minimal and credible sources. Several 
fake messages keep circulating on social media, which can be misleading and anxiety-provoking. ${ }^{5}$ For people on the edge of anxiety, constant hammering of news in media can be highly damaging and stressful. The high decibel noise generated by the media might inflate the size and gravity of the problem and might further add to the anxiety and confusion among high-risk individuals with fragile mental health.

Finding time for oneself: The importance of taking time out for oneself to introspect and have a break for inward-looking can significantly help in recharging the depleted sense of wellbeing. Music, dance, long walks or just sitting in silence can elevate the mood and clear the negativity. Little steps go a long way. Taking time to practice meditation and yoga is beneficial because exercise, yoga and meditation release endorphins which are feel-good hormones. Eating healthy food and getting enough rest to keep up with the daily challenges is also crucial.

Division of chores at home: Communication is key, discuss with your spouse about chore division. Don't expect your partner to understand what you want them to do and expect them to do it. Avoid unnecessary tensions and arguments, by chalking out a plan as to who does what.

Taking time out for kids amidst your work from home: Striking a balance between work life and family life is a challenging task. COVID-19 has grounded the working individuals, and the prolonged lockdowns have made everyone at home busy doing their work from home. The men are doing business over the internet using their laptops; wives are doing their jobs online and also taking care of the household chores. The children and students spend long stressful hours online staring at their mobile and computer screens for up to 8 hours a day. Alongside one's own office work, working wives also have to take care of kids' schoolwork, online working beside their usual household works. The stress from job mixes with the household stress and this leads to frustration and anger. Balancing the home and office life for most people seems very difficult as in online work atmosphere, the work hours have stretched beyond eight hours a day. Stressbusters such as taking time out with kids, taking a short break from work, helping the kids in their schoolwork, or doing some unusual activity such as gardening, cooking, or painting can help relieve stress. During the lockdown, the television showed gloomy reports of spread and deaths from corona pandemic and the entertainment programs had nearly stopped or played old episodes. Being locked at home for weeks at a stretch can be very stressful for most people and can be a lot worse and damaging for those who had to take pay cuts or suffer the loss of jobs. For such people, it is important to understand that the bad times, however tough, will pass, and it is important to hold tight against the tide. Family support in such times can be the best as people having negative thoughts realize that they have well-wishers who would pull them out of the bad patch.
The above suggestions, if practiced diligently, could alleviate stress, and keep one from slipping into despair. Transitions are complex, but being able to bring positive changes to routine, and lifestyle can be life changing and is warranted for the current situation.

\section{MEASURES AND POLICIES TO CURB SUI- CIDES}

Prevention of suicides calls for a multipronged strategy and needs careful planning. ${ }^{6}$ Several organizations have initiated zero suicide rates in their sphere of influence. The trick in preventing suicides is a timely detection in the individuals' behavioral patterns at higher risks for suicide and getting them connected to the resources promptly. What measures could be taken as an agency or by a service provider during the COVID situation to prevent the vulnerable population from slipping through the cracks?

Telehealth services for behavioural health have been in demand, and research from the University of Zurich has demonstrated equal improvement in depression for patients receiving face-to-face services vs online medical consultation. ${ }^{7}$ With the video calling technology provided by skype and Whatsapp doctors in India have started providing medical consultations over the phone. This telehealth is easily accessible and is cost-effective too. During the social distancing norms of Corona times, reaching out to the doctors is very easy and safe. Big hospital chains like Apollo and Fortis in metro cities have floated care packages for COVID patients. This saves patients from the travel requirements and wait for hours in the clinics to see their doctors. Increasing telehealth services, especially in the time of COVID-19 Pandemic, where restrictions have limited the easy accessibility to in-person visits, can facilitate contact between providers and the patients who need assistance. It is a known fact that in cases of suicide prevention, a timely call or a personal contact can often mitigate disaster. Suicide helplines strive to provide that pause and the succor that brings a person from the verge of committing suicide.

Research has shown a relationship between timely interventions and prevention of suicides by potential individuals. At every follow-up visit, clinicians and prescribers need to be vigilant regarding patients changing mental status and take appropriate measures to address the immediate issues. A systematic raising of a red flag for all potential suicide candidates can alert the care providers to be extra vigilant and save lives. Filtering the patients running high risks of committing suicide and then planning and customizing survival and safety strategies for each one of them is central to any prevention project. Since each individual is different, trying to know the warning signs, their support system, and developing coping strategies, and having patients verbalize them 
at each visit, have proven to mitigate the risk for suicide. ${ }^{8}$ Circumstances may change rapidly, updating the coping strategies, and reviewing with patients is paramount. One of the most crucial aspects of preventing suicide is restricting access to means. The importance of providing means restriction counselling cannot be emphasized enough. Ensuring safe storage of firearms reduces the risk for suicide. Personal or family firearms and prescription pills are most commonly used for committing suicide. The family members must keep a close watch on the presence of such drugs or firearms in the home and must take steps to safely lock them away out of reach of such vulnerable individuals. Firearms must be taken away and deposited safely and ammunition must be hidden away to prevent its use. In India, the gun control and license laws are very stringent, and family members must ensure surrender of such firearms. Censor orders on such individuals also might come in handy where their licenses and firearms might be suspended for extended periods to avoid any accidental mishap. ${ }^{9}$ In the case of prescription drugs such as sleeping pills or other scheduled drugs, utmost care must be exercised to keep them stored away out of reach of such individuals. Due diligence should be exercised in ensuring that patients are not stockpiling their pills, especially medicines such as those prescribed for combating anxiety. Stockpiling of such medicines must be prevented by selling additional vials only on production of the empty vials. Stringent laws for the pharmacies can help in doing this. Anxiety medicines such as diazepines are commonly used for suicide by overdosing on the same. ${ }^{10}$ Individuals with a high risk of suicide should not be left alone or allowed access to cleaning liquids and disinfectants or toilet cleaners as such harmful products are also ingested by many to commit suicide. Also, bringing awareness that they do not have to suffer abuse from their spouses, partners, or family members and seek help gives them a sense of security and hope. Often, patients might not know of the resources available to them, causing dejection and abandonment or helplessness, which makes them more vulnerable to harm. Therefore, it is essential to guide them about the availability of such handy resources. Patients should be provided with resources and contact information for a suicide hotline, domestic violence hotline, and for those needing assistance with crisis related to substance use. Creating fliers, tear-offs, handouts, cards with crisis information goes a long way and creates a safety plan, as those with the above crisis are vulnerable to suicide. According to research studies, public awareness campaigns have proven to be successful and beneficial in reducing the suicide rate among men. ${ }^{11}$ Other possible ways of potentially reducing risk for suicide is decreasing wait time on a suicide hotline, by increasing the number of counsellors available, for answering and providing comfort and reassurance when a person in need is trying to reach out for help. The individual at risk just needs a few words of compassion and expression that someone cares, at their weak moment. Caring and sup- portive words could uplift the spirits and may change one's decision about putting an end to life, preventing the dreadful from happening. Support of family is most important to look out for any remote and faint indications of elevated risk of suicide for individuals. They are checking every so often by visiting them, calling them, or even playing a video call for those who have the technological option.

In an event of failure of a patient to keep appointments, either in person, a video session or a phone session, leaving a message indicating their missed appointment and provider's intent to follow up soon gives a sense of hope and security. Especially for those at increased risk for suicide, making a caring contact call before the end of the week, assigning a simple task or having them come up with a small project to do over the weekend, gives them an activity to engage in. Follow up calls to check on the patients' wellbeing gives them a feeling of being cared for. Patients not reachable by phone messages by post or electronic text messages work equally well in ameliorating these patients' moods. A randomized control study conducted by Motto demonstrated that those who received caring letters intervention had a lower rate of suicide and suicide death than those who did not receive the caring letters. ${ }^{12}$

Failure to reach patients with repeated attempts should not be taken lightly. Requesting a wellness check by police can be a reasonable option. Other options such as sending a caring contact card indicating you are there for them if they need you and that you care about them. Any effort made to prevent someone from taking their life is, of course, a life saved.

Another intervention that is worth considering is a collaboration of behavioral health and primary care. Most individuals approach their primary care for depression, anxiety and suicidal ideations; primary care can evaluate and assess risk, initiate medications, and develop prevention strategies, and could refer them to behavioral health for further management. ${ }^{13}$ Grants and funding from government, private sectors and foundations, could be of immense help in implementing emergency response for suicide prevention. Looking into the possibility of aiding with housing and employment could mitigate the risk factors for suicide and reduce the number of suicides. The need of the hour is to press the government health infrastructure to work closely with organizations engaged in mental healthcare to formulate policies and steps to prevent cases of suicide. Locally, for the needy who are struggling to make ends meet due to a job loss and going through difficulty keeping up with basic needs, such as food, or medications, use of wrap-around funds by an agency is a plausible option.

\section{CONCLUSION}

COVID-19 Pandemic is a rapidly evolving situation with a lot of uncertainty. In a crisis like this, keeping up with the at- 
tendant demands and challenges demonstrates one's strength and resilience. These challenges call for drastic measures to combat and provide for the required changes. We have to be able to provide education and necessary tools to the patients that need assistance and make them aware that suicide is not the solution to their problems, and there are better ways to cope. We have to work closely with our fragile patient population, to provide them with the required support in developing more meaningful solutions and strategies that can keep them from sliding downhill. Suppose we can change their negative perception into believing that there is light at the end of the tunnel, and instill hope. In that case, we are making significant progress towards suicide prevention and zero suicide. Behavioural health organizations, Public health and government, should work in collaboration to assist those in dire need of help, in these trying times.

There are no cutting corners when it comes to patient care and safety. Many times, when we look in retrospect, following the loss of a patient to suicide, we ponder as to whether a certain intervention at the right time would have saved a patient's life? Exercising due diligence is of utmost importance, and the necessary steps can be taken even if there is the slightest hint that the patient has the potential to harm himself. Every effort should be made to support and protect our patient population, and there should not be any stone unturned because it is someone's life at stake. Risk reduction meetings are beneficial and provide valuable information regarding areas for improvement, which need to be followed through. With combined and persistent efforts, catastrophe can be averted from happening. At the community level, support groups are needed to be put in place to provide counseling to those at risk of suicide and achieve minimal or zero suicide rates. The pandemic has changed things like never before, and we as a community have to come to terms with the new normal. One needs to develop a positive attitude towards life and be thankful for every day and instill such attitude in our patient population.

Author's contribution: All the authors have equally contributed in preparing this document. Namita Rath and Vishal Sood conceived the paper's idea and put the base together while Alok Kumar Sahai did the final compilation, referencing and proofing of the paper.

\section{ACKNOWLEDGEMENT}

Authors acknowledge the immense help received from the anonymous reviewer for giving valuable suggestions for im- provement. The authors are grateful to all the scholars whose articles are cited and included in references of this article. The authors are also thankful to authors/editors/publishers of all those articles, journals and books from where the literature for this article has been reviewed and discussed.

\section{Conflict of Interest: Nil}

Funding Sources: Nil.

\section{REFERENCES}

1. Gunnell D, Appleby L, Arensman E, Hawton K, John A, Kapur $\mathrm{N}$, et al. Suicide risk and prevention during the COVID-19 pandemic. Lancet Psychiatry 2020;7(6):468-471

2. Windfuhr K, Kapur N. Suicide and mental illness: A clinical review of 15 years findings from the UK National Confidential Inquiry into Suicide. Br Med Bull 2011;100(1):101-21.

3. Tighe J, Nicholas J, Shand F, Christensen H. Efficacy of Acceptance and Commitment Therapy in Reducing Suicidal Ideation and Deliberate Self-Harm: Systematic Review. JMIR Ment Heal 2018;5(2): e10732.

4. Lambert G, Reid C, Kaye D, Jennings G, Esler M. Effect of sunlight and season on serotonin turnover in the brain. Lancet 2002;360(9348):1840-1842.

5. Gao J, Zheng P, Jia Y, Chen H, Mao Y, Chen S, et al. Mental health problems and social media exposure during COVID-19 outbreak. PLoS One 2020;15(4): e0231924.

6. Pisani AR, Murrie DC, Silverman MM. Reformulating suicide risk formulation: From prediction to prevention. Acad Psychiatry 2016;40(4):623-629.

7. McWilliams J, Myers K. Telemental health. Tech Adolesc Mental Health 2018:171-185.

8. Bryan CJ, Mintz J, Clemans TA, Leeson B, Burch TS, Williams SR, et al. Effect of crisis response planning vs. contracts for safety on suicide risk in U.S. Army Soldiers: A randomized clinical trial. J Affect Disord 2017; 212:64-72.

9. Rodríguez Andrés A, Hempstead K. Gun control and suicide: The impact of state firearm regulations in the United States, 1995-2004. Health Policy (New York). 2011;101(1):95-103.

10. Agrawal R, Verma S, Halappanavar M. Measuring the Effectiveness of Benzodiazepine Prescriptions Control in Community Setting Using Prescription Drug Monitoring Program (PDMP). Community Ment Health J. 2020. doi: 10.1007/s10597-02000686-5. Online ahead of print

11. Matsubayashi T, Ueda M, Sawada Y. The effect of public awareness campaigns on suicides: Evidence from Nagoya, Japan. J Affect Disord 2014;152-154:526-529.

12. Motto JA, Bostrom AG. A randomized controlled trial of postcrisis suicide prevention. Psychiatr Serv 2001;52(6):828-833.

13. Okolie C, Dennis M, Simon Thomas E, John A. A systematic review of interventions to prevent suicidal behaviors and reduce suicidal ideation in older people. Int Psychogeria 2017;29(11):1801-1824. 